\title{
Parametric Design Learning Strategies in the Context of Architectural Design Remote Teaching
}

\author{
Angélica Paiva Ponzio¹, Mario Guidoux Gonzaga ${ }^{1}$, Marina Pires De Castro \\ Aguiar Vale ${ }^{1}$, Underléa Miotto Bruscato ${ }^{1}$, William Mog $^{1}$ \\ ${ }^{1}$ Universidade Federal do Rio Grande do Sul, Brasil \\ angelica.ponzio@gmail.com \\ guidoux.gonzaga@gmail.com \\ marinapires.design@gmail.com \\ arq.leiab@gmail.com \\ williammog@hotmail.com
}

\begin{abstract}
This article aims to demonstrate how a theoretical-didactic model and its respective teaching strategies for algorithmic-parametric logic can act as potential elements of innovation in the architectural design process. Based on the theories of parametric design thinking by Oxman (2017) and the studies by Woodbury (2010) and Romcy (2017), such strategies are based on the principle that algorithmic logic can be understood, in certain circumstances, as a procedural framework and not just an instrumental one. It will also be discussed how the situation of remote learning in the face of the COVID-19 crisis brought about the use of virtual teaching tools as an increment of the learning process.
\end{abstract}

Keywords: Architectural teaching, computational design, parametric design thinking, design process, algorithmic design.

\section{Introdução}

A chamada era digital tem provocado importantes transformações no ensino e na prática da arquitetura. Nesse sentido, as ferramentas digitais têm cada vez mais se afastado das atribuições de meros instrumentos de representação, passando a atuar como definidoras do processo projetual (Natividade, 2011). De tal forma, autores como Caetano, Santos e Leitão (2020) destacam o conceito de "projeto computacional", ou "computational design" assumindo um papel bem diferente do "projeto digital", ou "digital design" - enquanto este 
último se refere ao uso de ferramentas computacionais no processo projetual, o primeiro é entendido como o uso de computação, analógica ou digital, no desenvolvimento de processos projetuais. No âmbito acadêmico, fomentando um crescente debate sobre metodologias emergentes no ensino do projeto, educadores e pesquisadores têm se direcionado a estudar os diferentes processos envolvidos abrangendo níveis de interação entre o projetista e os modelos digitais. No caso da América Latina, e mais especificamente no Brasil, autores como Romcy (2017), Celani e Veloso (2017) apontam a importância da implementação de modelos cognitivos que incluam o aprendizado digital no processo projetual no currículo de ensino da arquitetura. Visando contribuir com este debate, o presente artigo busca apresentar estratégias abordadas por um modelo didático-teórico voltado a uma disciplina de representação gráfica digital do segundo ano de ensino de graduação em arquitetura. Sua fundamentação teórica se embasa nos estudos realizados no âmbito de uma Pesquisa Teórica de Referência sobre inovação no processo projetual arquitetônico e do respectivo Grupo de Pesquisa do Cnpq. Partindo do princípio de que a lógica algorítmica pode ser entendida, em determinadas circunstâncias, como referencial processual e não meramente instrumental, a metodologia abordada enfatiza - por meio de um enfoque teórico-prático -, o emprego de exercícios analógicos com uma progressiva transição para meios digitais. Ao delimitar diferentes etapas de instrumentalização da modelagem paramétrica, as estratégias propostas objetivam assim evitar a mimetização de códigos prontos, favorecendo ao mesmo tempo o entendimento do raciocínio lógico e seu potencial emprego durante as fases iniciais de ideação e desenvolvimento de um projeto. Como consequência, espera-se promover nos discentes uma certa autonomia de como e quando relacionar estes conhecimentos no processo projetual.

Primeiramente será feita uma contextualização do ambiente de trabalho e da disciplina, incluindo a recente realidade do trabalho remoto para, na sequência, descrever a metodologia de ensino com suas respectivas etapas visando o aprendizado de saberes específicos. Finalmente, na conclusão, serão apresentados os resultados obtidos sob o ponto de vista didático e os possíveis encaminhamentos futuros.

\section{Antecedentes - Representação Gráfica III}

A disciplina aqui apresentada faz parte de um grupo de três disciplinas responsáveis pelo ensino da representação arquitetônica digital de um curso de graduação em arquitetura, a saber: Representação Gráfica I (RG-I), em que 
são abordadas normas de desenho técnico partindo de programas CAD, finalizando com uma introdução à modelagem em BIM; Representação Gráfica II (RG-II), focada exclusivamente na modelagem em BIM; e, por fim, Representação Gráfica III (RG-III) na qual, a partir de 2018, uma nova súmula foi estabelecida abordando novos recursos digitais de desenho paramétrico, técnicas de prototipagem e visualização. Entre seus objetivos, encontra-se propiciar o exercício da análise crítica projetual através de exercícios de caráter analítico-exploratórios como contribuição para o entendimento do objeto arquitetônico. Nesse escopo destaca-se uma introdução ao pensamento computacional, onde parte-se do ensino de um processo e não de uma mera ferramenta tecnológica. A estratégia didática busca ainda, através da capacitação de conhecimentos de programação algorítmico-paramétricos, estabelecer as bases para um possível diálogo com as disciplinas práticas de projeto. Em um processo de aprimoramento crescente, a disciplina apresenta, após uma introdução teórica, exercícios analógicos iniciais para o entendimento da lógica algorítmica. Na sequência são apresentados dois blocos principais de tutoriais visando o ensino de saberes específicos preparatórios, focados no estudo da forma e serialização. O semestre finaliza com o projeto em grupo de pequenos objetos arquitetônicos com variações paramétricas, elaborados através da montagem de um processo baseado em regras e instruções que são factíveis de serem avaliadas, revisadas e aprimoradas. Em $2020^{1}$, e diante da nova realidade ocasionada pela pandemia da Covid-19, foi realizada uma adequação ao chamado Ensino Remoto Emergencial. Neste panorama, a plataforma Miro associada ao Microsoft Teams se mostraram fundamentais ao reproduzir o ambiente de sala de aula e possibilitando, ainda, a interação no contexto da didática proposta, que será apresentada a seguir.

\section{$3 \quad$ Metodologia}

\subsection{O Ensino Remoto Emergencial}

A pandemia da Covid-19 impactou as nossas vidas de forma generalizada, fazendo com que vários hábitos fossem reformulados ou reinventados. Com a prática do ensino de graduação em arquitetura não foi diferente. Diante desta

\footnotetext{
${ }^{1}$ Turmas dos professores Angélica Paiva Ponzio, Underléa Miotto Bruscato, William Mog e estagiários docentes Marina Aguiar Vale e Mario Guidoux Gonzaga.
} 
nova realidade, formas e estratégias de construção do conhecimento foram (re)elaboradas para o contexto pandêmico que, apesar das limitações impostas, trouxe importantes oportunidades. Dessa maneira, e embasados nos estudos realizados no âmbito de uma Pesquisa Teórica de Referência e seu respectivo Grupo de Pesquisa, a equipe composta por professores, estagiários docentes e monitores aprimorou a estratégia didática buscando minimizar as limitações a partir da maximização das potencialidades. O modelo remoto (ERE) aplicado, em oposição ao EAD, foi elaborado então a partir de um levantamento de ferramentas virtuais de apoio passíveis de serem utilizadas através da avaliação de potencialidades e adequação aos exercícios, em um processo descrito a seguir.

\subsection{As ferramentas disponibilizadas}

Com a necessidade do ensino remoto, adicionou-se uma nova camada de complexidade ao ensino de softwares: como transmitir conhecimento e manter engajados em trabalhos em grupo alunos que estão fisicamente afastados há meses? A solução encontrada foi a combinação de ferramentas complementares que auxiliaram em aspectos fundamentais da disciplina. A comunicação e transmissão das aulas deu-se a partir do sistema Microsoft Teams. Adotado pela Universidade como plataforma oficial de videoconferências, a plataforma permitiu a gravação das aulas para a revisão/assistência de conteúdos de modo assíncrono, além de possibilitar a criação de salas de conversa independentes. Somado a isso, o aplicativo Miro $^{2}$ desempenhou papel fundamental na interação síncrona e assíncrona. Entendido não apenas como um quadro-negro virtual, mas também como espaço análogo ao ambiente de aula físico, permitiu não apenas que os estudantes compartilhassem uma prancheta virtual e colaborassem na montagem de painéis e exercícios durante a aula, mas também estimulou 0 contato de grupos de maneira assíncrona. Complementar a isso, os arquivos de apoio para as aulas e as gravações realizadas durante os encontros ficaram disponíveis para os estudantes durante o semestre na plataforma Moodle, utilizada pela Universidade desde antes do ERE.

\subsection{As Estratégias}

No primeiro semestre de 2020, a disciplina dividiu-se em três instâncias de interação, conduzindo a um aprofundamento gradual de complexidade no

\footnotetext{
${ }^{2}$ Disponível gratuitamente em https://www.miro.com
} 
âmbito do desenho paramétrico. A primeira instância destinou-se à sensibilização e à introdução ao pensamento paramétrico e algorítmico (Oxman, 2017; Caetano, Santos e Leitão, 2019) por intermédio de aulas teóricas seguidas de cinco exercícios analógicos em ambiente virtual. Buscouse ainda sugerir aqui, através de exercícios com de diagramas de nós, uma possível estratégia para desenvolvimento de um processo projetual. A segunda instância visou a instrumentalização da ferramenta Rhino/Grasshopper e a familiarização com os componentes do programa, valendo-se de modelos de referência. $\mathrm{O}$ objetivo aqui não foi o de apenas gerar formas complexas, mas sim explorar o pensamento algorítmico em capacidades de serialização e otimização formal associadas à variação paramétrica. Por fim, os estudantes foram conduzidos em grupos à elaboração de uma família modular paramétrica de objetos arquitetônico/expositivos, localizada em um parque da cidade que, respeitando os limites da Covid-19, contemplasse estratégias associadas às normas de distanciamento social.

\section{Sensibilização (aulas 1-3) - exercícios analógicos}

Nesta etapa, após aulas teóricas introdutórias sobre o pensamento computacional, deu-se início uma série de cinco exercícios analógicos de iniciação ao pensamento algorítmico-paramétrico. No ambiente colaborativo de "canvas infinito" propiciado pela ferramenta virtual Miro, ferramentas de design thinking disponibilizadas na plataforma foram usadas para uma construção do entendimento do pensamento computacional. Dessa maneira, no canvas virtual coletivo os alunos foram conduzidos em grupos a desmembrar sequências de ações diárias (instruções) por meio de diagramas de nós, no intuito de, posteriormente, aplicar esta estratégia ao pensamento projetual arquitetônico. Para essa transposição, por sua vez, foram fornecidos para análise projetos de pequenas edificações de formas simples, como 0 cubo, com alterações booleanas. Nesse momento os alunos deveriam reproduzir a lógica da montagem volumétrica, ainda de maneira analógica no Miro, partindo de uma descrição referenciada no espaço. Esse exercício também serviu para nortear a apresentação inicial do funcionamento do Grasshopper através de sua implementação digital.

Esta etapa valeu-se, portanto, do entendimento da lógica sequencial algorítmica e de seu emprego em áreas além da estritamente computacional. Entende-se que as possibilidades de transposição exercitadas visam a demonstração de seu emprego não apenas em nossas tarefas diárias, mas também no campo projetual arquitetônico, facilitando o entendimento do 


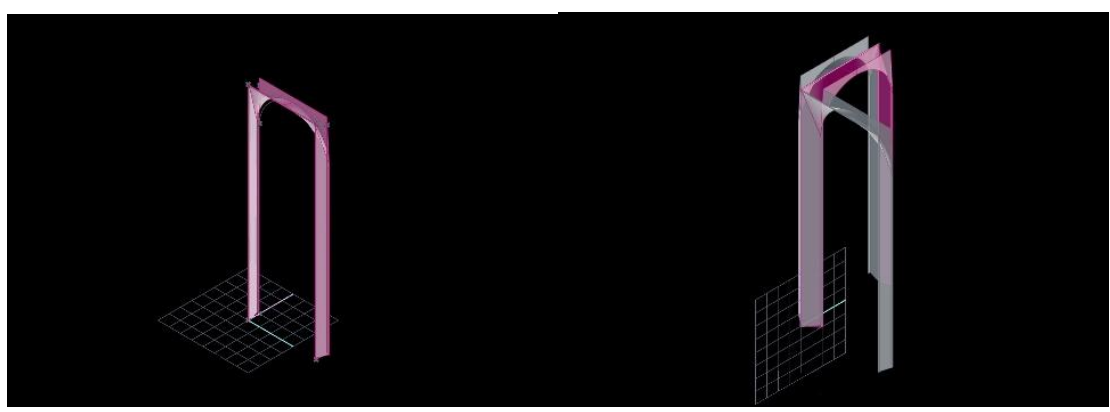

Figura 2. Modelagem dos arcos. Fonte: RG-III, 2020.

A segunda parte do exercício foi dividida em dois momentos: ao modelar o arco de canto, no qual existe uma distorção na largura de um dos lados do arco, foram explorados os conceitos de rotação e espelhamento, aprofundando o entendimento sobre planos e vetores. No segundo momento, a noção de modularidade foi explorada multiplicando os arcos em um lado do edifício e, logo após, criando cópias rotacionadas de cada uma das quatro fachadas, finalizando a envoltória do Itamaraty (Fig. 3).

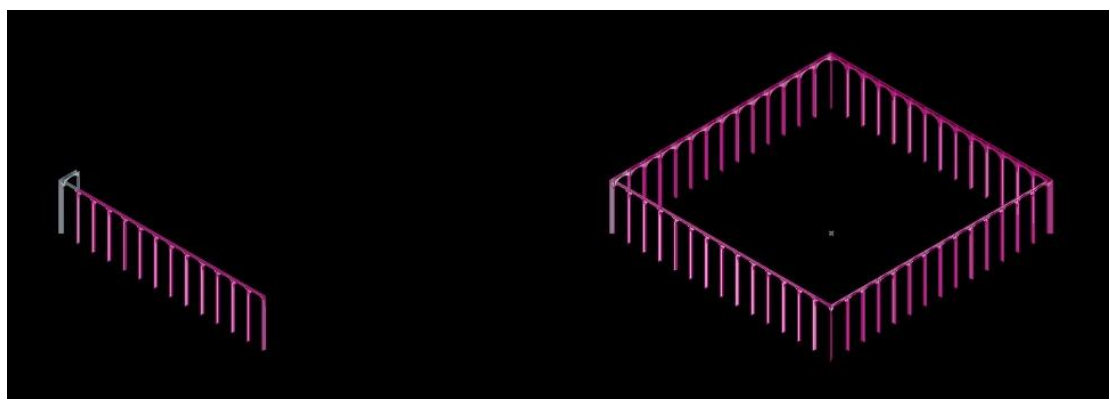

Figura 3. Seriação e modularidade. Fonte: RG-III, 2020.

O domínio da seriação permitiu aos estudantes aprofundar o trabalho com listas de objetos ao desenhar a fachada do volume envidraçado do Palácio (Fig. 4). Para as esquadrias, utilizou-se da seriação simples de elementos verticais e da variação do espaçamento dos elementos horizontais, conforme a altura das lajes internas, introduzindo ferramentas de seleção a partir de padrões booleanos (cull-pattern). 


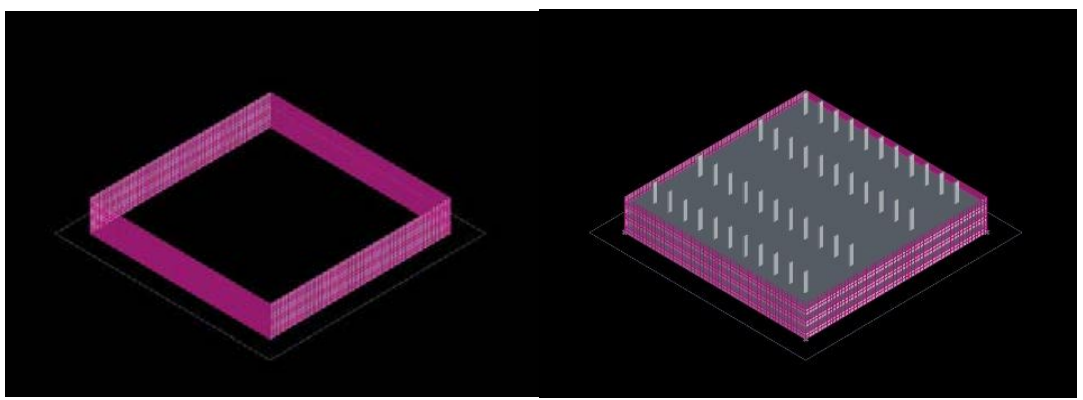

Figura 4. Seriação avançada na fachada e estrutura. Fonte: RG-III, 2020.

Finalmente, foi possível modelar a estrutura portante do volume interno combinando o conhecimento de todas as etapas: para as lajes, aproveitou-se o padrão de seleção das esquadrias, enquanto para os pilares a modelagem utilizou seriação e espelhamento (Fig. 5).

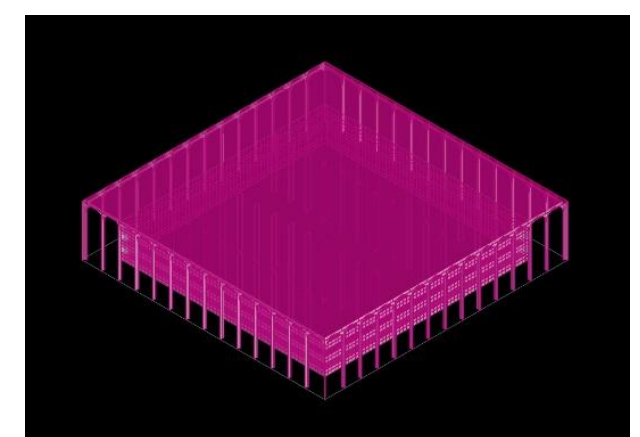

Figura 5. Modelo finalizado. Fonte: RG-III, 2020.

Como uma demonstração da capacidade deste tipo de software, foi apresentada ainda, ao final do exercício, uma modificação nos parâmetros de espaçamento dos arcos aludindo ao projeto de Niemeyer para a sede da Editora Mondadori, em Milão.

\section{Aplicação (aulas 9-16) - Aplicação dos conhecimentos - O Brique}



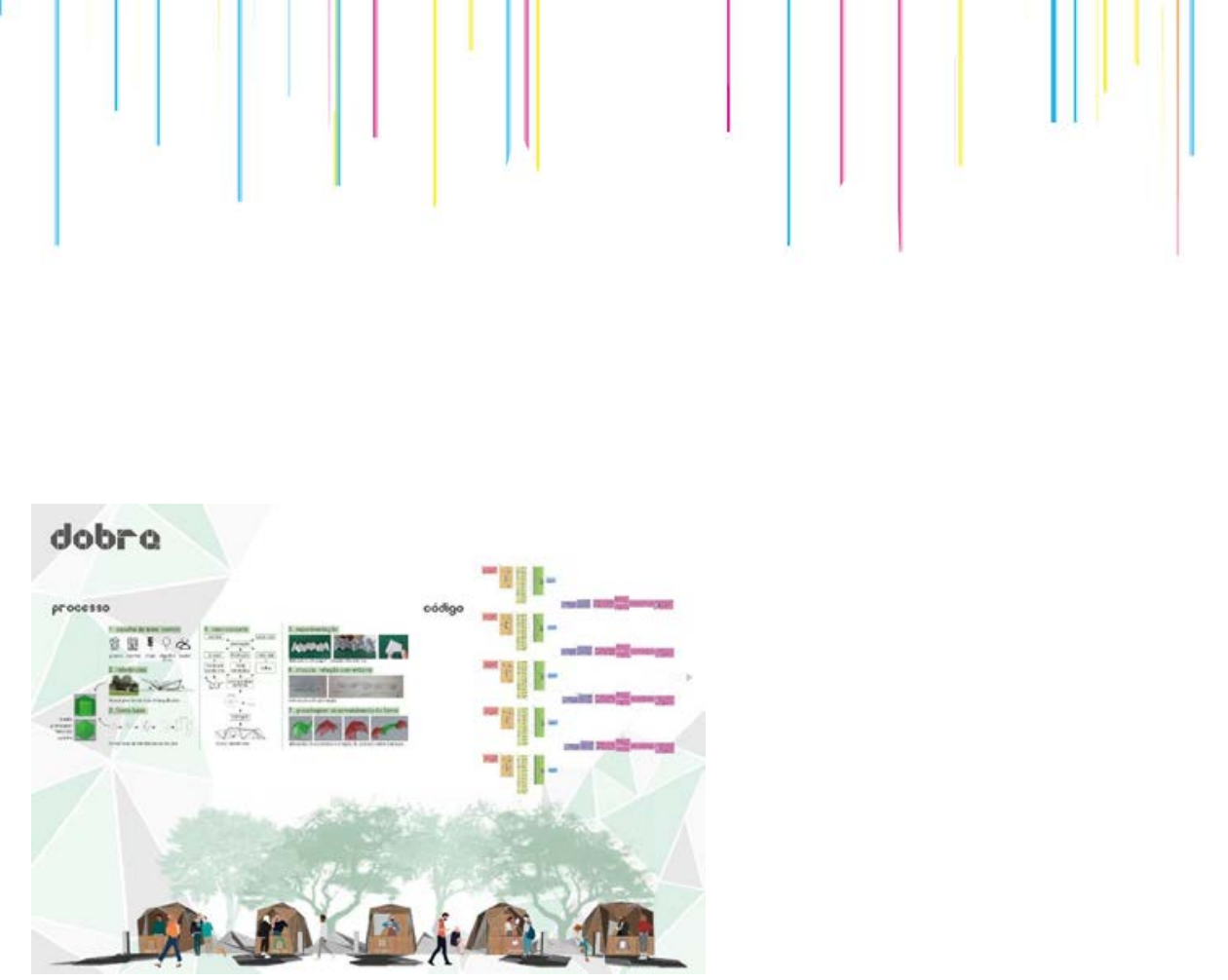

Figura 07. Projeto Dobra. Fonte: Carolina Mattiello, Eduarda Bittencourt, Gabriela de Souza, Jéssica Rosa, Paola Osterkamp.

Após a introdução e a instrumentalização dos alunos, foi proposto um exercício final que envolveu a aplicação da lógica paramétrica no processo projetual. A temática proposta envolveu o desenvolvimento de um grupo de módulos parametrizáveis para o espaço de uma feira de parque, o Brique do Parque da Redenção. O exercício foi desenvolvido em equipes, em que, a partir de um conceito comum, deveria-se elaborar uma forma base geral (algoritmo base) passível de variações paramétricas particulares propostas individualmente. $\mathrm{Na}$ etapa final, os grupos apresentariam o conjunto das propostas ambientadas no local levando em consideração o distanciamento social e o contexto da pandemia. Sugeriu-se ainda a utilização do "urbanismo tático" (WRI, 2018) como estratégia de implementação do programa de necessidades proposto por cada equipe.

O desenvolvimento do exercício ocorreu a partir de uma introdução teórica e temática sobre o local e desenrolou-se em quatro aulas práticas além da apresentação final. Organizados em grupos de cinco alunos, estes desenvolveram os trabalhos em duas etapas abrangendo a plataforma Miro e Grasshopper. A primeira etapa partiu da definição de um conceito, temática e respectivo programa de necessidades do conjunto de unidades de cada grupo. Ao optar por um de dois tipos possíveis de implantação - linear ou radial -, os estudantes deveriam planejar um módulo base parametrizável, de acordo com a temática definida. Como resultado desta etapa, destaca-se a apresentação do conceito e temática com seus respectivos produtos, assim como um prélançamento da disposição do conjunto e croquis iniciais de geração da forma. As apresentações se deram na plataforma Miro. A segunda etapa partiu para o desenvolvimento do código no Grasshopper e assessoramentos complementares no ambiente do Miro. Foram lançados os primeiros códigos, 
auxiliados por diferentes estratégias de desenvolvimento, como diagramas de nós, croquis e maquetes físicas de estudo. Após a definição do código base, os grupos testaram as variações paramétricas individuais. Neste ponto houve ainda um painel intermediário, visando a apresentação de uma imagem do código geral e de uma imagem de cada uma das variações paramétricas. Ao final do semestre todo o material digital (pranchas de apresentação em PDF e código no Grasshopper) foi entregue no repositório virtual Moodle da disciplina. A avaliação de cada equipe e de cada aluno ocorreu em função da realização dos exercícios e trabalhos propostos e entregues, e foi ponderada pelos critérios de domínio e articulação dos conteúdos; competência e compreensão no uso da ferramenta Grasshopper e da sua lógica de modelagem; uso de recursos gráficos para representação do código; qualidade das ambientações, diagramação, conteúdo gráfico, tratamento das imagens.

\section{Encaminhamentos futuros}

A disciplina aqui apresentada tem buscado desenvolver uma metodologia que visa a fomentar uma lacuna quanto ao ensino de novas tecnologias que contribuam para o entendimento do objeto arquitetônico e de seus processos de formação. Dessa maneira, demonstrou-se aqui não somente como a lógica algorítmica pode atuar no processo de projeto, mas também como exemplos da cultura arquitetônica moderna e contemporânea podem ser empregados no intuito de explorar tais tecnologias enquanto parte de uma didática. Demonstrou-se também como a sala de aula, ainda que remota, pode abarcar assuntos atuais de interesse comum à sociedade. Mais ainda, como o ambiente remoto pode contribuir na formação acadêmica e profissional através do contato com novas ferramentas, que vieram a agregar-se positivamente à dinâmica de interação em sala de aula. Finalmente, salienta-se ainda o potencial de aplicação do pensamento algorítmico em complementação e/ou em conjunto com demais disciplinas de projeto e software BIM, associadas ainda a técnicas construtivas, desempenho e conforto térmico, visando uma maior integração, interdisciplinaridade, e aprofundamento das tecnologias. Tem-se previsto ainda para este ano a apresentação na disciplina de técnicas e ferramentas inovadoras de Inteligência Artificial (IA), Aprendizado de Máquina (Machine Learning) e Aprendizado Profundo (Deep Learning) e seu emprego em potencial no processo de projeto. 


\section{Referências}

Caetano, I., Santos, L., \& Leitão, A. (2020). Computational design in architecture: Defining parametric, generative, and algorithmic design, Frontiers of Architectural Research, volume 9, Issue 2, pp. 287-300. https://doi.org/10.1016/j.foar.2019.12.008

Celani, G., \& Veloso, P. (2017) The intersection of theory and technology: computational concepts applied to architectural design since the late 1980s. In Frontiers of Science and Technology: Automation, Sustainability, Digital Fabrication - Selected Extended Papers of the 7th Brazilian-German Conference. (pp. 139-151). De Gruyter.

Natividade, V. G. (2011). Fraturas metodológicas nas arquiteturas digitais, Dissertação de Mestrado, Universidade de São Paulo.

Oxman, R. (2017) Thinking difference: theories and models of parametric design thinking. Design Studies, volume 52, pp. 4-39.

Romcy, N. M. E. S. (2017). Abordagem Paramétrica e Ensino de Projeto: Proposição de Diretrizes Metodológicas, considerando estratégias curriculares e o atelier de projeto. Tese (Doutorado em Arquitetura e Urbanismo). Universidade Federal do Rio Grande do Norte.

Woodbury, R. (2010). Elements of Parametric Design. Routledge.

WRI Brasil. (2018). O poder de transformação do urbanismo tático. Acessado em 28 de julho de 2021, a partir de https://wribrasil.org.br 